\title{
Extraction Of Feature Lines On Surface Meshes Based On Discrete Morse Theory
}

\author{
J. Sahner and B. Weber and S. Prohaska and H. Lamecker, Zuse Institute, Berlin
}

\begin{abstract}
We present an approach for extracting extremal feature lines of scalar indicators on surface meshes, based on discrete Morse Theory. By computing initial Morse-Smale complexes of the scalar indicators of the mesh, we obtain a candidate set of extremal feature lines of the surface. A hierarchy of Morse-Smale complexes is computed by prioritizing feature lines according to a novel criterion and applying a cancellation procedure that allows us to select the most significant lines. Given the scalar indicators on the vertices of the mesh, the presented feature line extraction scheme is interpolation free and needs no derivative estimates. The technique is insensitive to noise and depends only on one parameter: the feature significance. We use the technique to extract surface features yielding impressive, non photorealistic images.
\end{abstract}

Categories and Subject Descriptors (according to ACM CCS): I.3.3 [Computer Graphics]: Line and Curve Generation I.4.6 [Computer Graphics]: Feature Detection I.4.7 [Computer Graphics]: Feature Measurement

\section{Introduction}

The extraction of salient features is a crucial task in visualization, and surface meshes are a common digital representation of objects to be visualized. In non-photorealistic rendering, for instance, the goal is to depict a surface with a sparse set of characteristic features only. Another important example is the registration and fusion of a number of shapes, e.g. for atlas generation in bio-medical applications, such that common features are identified correctly.

Most commonly, feature lines are defined as ridges and ravines of the surface, a notion of differential geometry including third orders of surface derivatives. Several highly evolved algorithms exist to extract feature lines based on this definition [Ebe96, OBS04, YBS05, YBYS07, HPW05, SF04]. As the stability of third derivatives is difficult to control, those approaches require the adaptation of a number of parameters on a per surface basis. This is especially unsatisfactory in registration applications, where a large number of surfaces has to be processed to establish an anatomic atlas.

In this paper we will undertake an alternative approach based on Morse Smale (MS) theory [Mil63, Ban70, EHZ03, Pas07,For98], which is an algebraic tool for measuring topological features of a function $f: M \rightarrow \mathbf{R}$ on a manifold $M$. A simplification strategy can be used to obtain a hierarchy of MS complexes giving rise to a natural discrimination of feature scales. We will present a modification of the original persistence simplification strategy that is capable of extracting extremal feature lines in the sense that the value of $f$ is significantly larger or smaller on the feature line than in its vicinity. This extremality property is satisfied by the 1-cells of the initial MS complex that contain the watersheds and watercourses [Soi99] as special cases.

Our main contributions are:

- We identify suitable feature indicator functions for extracting extremal feature lines (Sect. 3.1).

- We show that persistence simplification is not useful for extracting extremal feature lines (Sect. 3.3).

- We introduce a novel simplification strategy that preserves extremal feature lines (Sect. 3.4).

- MS based feature detection necessarily produces connected lines, which is not generally desired. We propose a method to alleviate this problem by discarding unwanted lines in a post-processing step (Sect. 3.5).

The main benefits of our method are:

- By using discrete Morse Theory, the extraction method does not require any derivative estimates.

- There is only one user-defined parameter in the method defining the level of significance of the resulting features.

- The method is insensitive to noise in the input fields (Sect. 4.1). 


\subsection{Previous Work}

Edelsbrunner et al. [EHZ03, BEHP04, Pas07] and Forman [For98, For02, Lew05] have presented extensions of classical Morse theory to triangulated surfaces. Those approaches have been used in various areas for as different tasks as function simplification that bundles topology and geometry [BEHP04], molecular docking applications [CCL03, $\left.\mathrm{NWB}^{*} 06\right]$, quadrangular surface remeshing using natural harmonics $\left[\mathrm{DBG}^{*} 06\right]$, and vortex and strain analysis of $3 D$ flow fields [SWTH07]. A closely linked subject is watershed segmentation, as the watersheds of a function are contained in the Morse-Smale complex [Soi99]. Mangan et al use [MW99] watershed segmentation on surface meshes for surface patchification. Those approaches strive to generate good patch layouts of the surface rather than extract feature lines. Recent advances introduce skeletons of a distance field function that is assembled of parts of the 1-cells of the complex [GMDP*07].

Several approaches for ridge line extraction on surfaces exist. Ohtake et al [OBS04], Yoshizawa et al [YBS05, YBYS07], Hildebrandt et al [HPW05] and Stylianou et al [SF04] present different approaches for controlling the involved third derivatives of the surface. While all those approaches yield impressive results, they suffer from the fact that a number of parameters has to be adjusted.

\section{Hierarchical Morse-Smale Complex}

A smooth function $f$ on a smooth manifold surface $M$ without boundary embedded in $\mathbf{R}^{3}$ is a Morse function if its Hessian has full rank at every critical point of $f$ where its gradient vanishes [Mil63,Ban70,PM82,EHZ03]. Gradient curves are maximal lines of $f$ that are everywhere tangent to the gradient. Gradient curves start and end at critical points $c$ of $f$. The stable and unstable manifolds $S(c)$ and $U(c)$ are the collections of all points that are part of a gradient curve ending at $c$ or originating from $c$, respectively. The stable and unstable manifolds of maxima and minima can be regarded as hills and valleys, respectively. Critical points that have both a nontrivial stable and unstable manifold are saddles. Both the collection of stable manifolds and the collection of unstable manifolds partition the surface, see Fig. 1. For Morse functions $f$, the intersection of both partitions is the Morse-Smale complex of $f$. This complex subdivides the domain into quads with alternating maximum, saddle, minimum, saddle, see Fig. 1d. The one dimensional arcs in the decomposition link maxima, minima and saddles and are called ascending (descending) 1-cells, if they connect saddles with maxima (minima). Descending (ascending) 1-cells separate hills (valleys) and constitute their boundary.

\subsection{Cancellation}

Pairwise cancellation of saddle/minimum or saddle/maximum can be performed to coarsen the initial complex, as long as the alternating quad property is maintained. A cancellation is called valid in this case. Fig. 1e

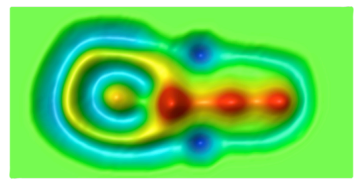

(a) Elevation profile.

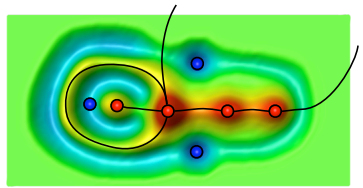

(c) Valleys and their borders.

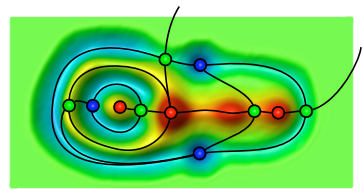

(e) Maximum cancellation.

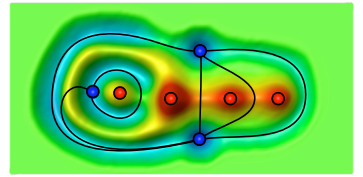

(b) Hills and their borders.

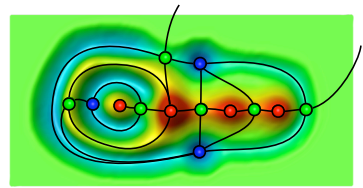

(d) Morse Smale complex.

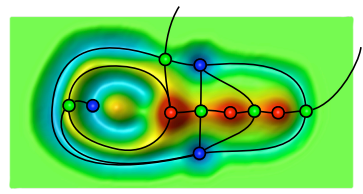

(f) Terminal maximum.
Figure 1: The elevation profile of a landscape as seen from above. Lines depict the partition of the landscape into hills, valleys and their intersection, the MS complex. Blue, red and green dots represent minima, maxima and saddles. Bottom row: simplifications of the MS complex by different types of cancellations and their influence on vanishing 1-cells.

illustrates how a saddle/maximum cancellation usually erases the descending 1-cell of the canceled saddle. The ascending 1-cell stays in the complex, as it still divides the unstable manifolds of the minima adjacent to the canceled saddle. In the special case of a terminal minimum or maximum, both the unstable and the stable manifolds of the saddle vanish, see Fig. 1f. By providing a sequence of valid cancellations, the complex can be coarsened, until no valid cancellation is left. In practice this is done by quantifying each saddle individually by a cancellation criterion. The cancellations are then performed in increasing order of the cancellation criterion.

\subsection{Discrete Computation of the MS Complex}

Based on Banchoff's extension of classical Morse theory to polyhedral surfaces [Ban70], Edelsbrunner et al [EHZ03] construct the MS complex for piecewise linear functions. The approach is refined by Bremer et al [BEHP04, Pas07].

Forman [For98, For02] generalizes Morse Theory to the more general category of combinatorial complexes. This generalization is a very strong theoretical foundation for simple and intuitive algorithms. Therefore we base our algorithms on this theory.

A function $f$ defined on the vertices of a triangular surface can be considered a Morse function if all vertex values are different. Lewiner et al [LLT03b, LLT03a, CCL03] show how to construct the discrete gradient vector field (also for non-Morse functions) as a pair of interlaced primal and dual 


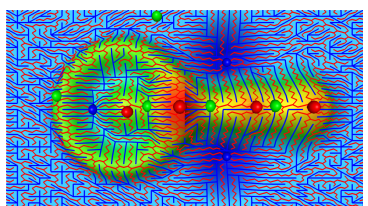

(a) Discrete gradient field

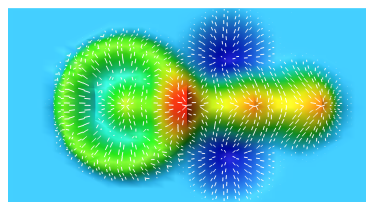

(b) Smooth gradient field
Figure 2: Forman's discrete gradient vector field of the height function of an elevation profile is shown with red maxima, green saddles and blue minima in (a). Red lines are gradient curves ascending to maxima, blue lines are gradient curves descending to minima. In (b) its smooth analogue is shown, being discontinuous and constant on triangles.

weighted spanning forests of the triangular surface that partition the surface into stable and unstable manifolds.

- The weighted primal graph $P G$ is the graph of vertices and edges of the surface mesh. Edges are assigned the mean value of the incident vertices.

- The vertices and edges of the dual graph $D G$ are the triangles and edges of the surface mesh, respectively. Triangles are assigned the mean value of the incident vertices.

The maxima of the dual graph serve as roots for a spanning forest representing stable manifolds. Edges are added in decreasing order. Similarly, minima of the primal graph serve as roots for a spanning forest representing unstable manifolds. Edges are added in increasing order, but only if they are not contained in the dual spanning forest. The critical elements of $f$ are the roots of the primal (minima) and dual (maxima) graph and the edges that are not contained in either spanning trees (saddles). Lewiner shows that the extracted locations of critical points correlate with the locations Banchoff describes [Ban70]. The spanning forests can be regarded as the union of all gradient curves on the mesh. Descending gradient curves are traced as concatenated edges in the primal spanning tree as paths to the root, ascending gradient curves as triangle strips in the dual spanning tree. See Fig. 2 for the discrete gradient vector field of the height function of an elevation profile.

The computation of the 1-cells can now be performed as in the smooth setting. For a saddle edge $e=\left(v_{1}, v_{2}\right)$ that is incident to the faces $f_{1}, f_{2}$, the stable manifolds are computed as decreasing paths in the primal spanning tree starting with $v_{1}, v_{2}$ respectively until the roots $m_{1}, m_{2}$ (that are minima) are reached. Analogously, unstable manifolds are traced as triangle strips in the dual spanning tree starting from $f_{1}, f_{2}$ respectively, until the maxima $M_{1}, M_{2}$ are reached.

\section{Discrete Feature Line Extraction Method}

\subsection{Scalar Feature Indicators}

On a smooth surface, the two principal curvatures $\kappa_{1} \geq \kappa_{2}$ measure the bent of the surface along the corresponding curvature directions. Therefore, the principal curvatures are indicators for surface specific feature lines. Whereas $\kappa_{1}$ is maximal along convex feature lines, $\kappa_{2}$ is minimal along concave feature lines. Curvedness, as introduced by Koenderink et al [KvD92], is defined by $C=\sqrt{\frac{1}{2}\left(\kappa_{1}^{2}+\kappa_{2}^{2}\right)}$. This measure is large whenever any principal curvature has a large absolute value, and hence measures convex and concave regions simultaneously. It is maximal along both convex and concave feature lines.

On a triangulated surface, $\kappa_{1}$ and $\kappa_{2}$, can be defined discretely for each vertex of the surface using the method described by Hildebrandt et al [HPW06].

\subsection{Morse-Smale Complex and Feature Lines}

Ascending 1-cells are maximal lines of scalar feature indicators, as they are lines of steepest ascent separating two valleys if the 1-cell is a watershed, or one valley, if the 1cell is connected to a terminal maximum, see Fig. 1f, and analogously for descending 1-cells. Depending on the scalar feature indicator, just the ascending $\left(\kappa_{1}, C\right)$, or descending $\left(\kappa_{2}\right)$ 1-cells are of interest. Not all of these 1-cells are necessarily features. This is due to the fact that noise adds additional saddles of small difference in function value, an effect known as oversegmentation in the field of watershed transformation [Soi99]. We aim at differentiating the 1-cells that are salient features from noise by applying a cancellation criterion that favors the concentration of scalar indicator value on 1-cells opposed to their vicinity.

\subsection{Persistence Cancellation}

The persistence [ELZ00] of a saddle $s$ is the least difference in function values compared to the cancellable extrema it is linked to. Cancelling saddles by increasing persistence realizes topological simplification by means of the least geometric change, see Bremer et al [BEHP04].

Persistence cancellation does not preserve feature lines as seen at the rocker arm surface in Fig. 3 and the ascending 1-cells of the initial MS complex of $\kappa_{1}$ containing the convex feature lines of interest. Persistence cancellation initially keeps this property in Fig. 3b. However, further cancellation destroys one of the most characteristic convex feature lines, and a line traversing a concave region is preferred, see Fig. 3c. The obvious reason for this is that the persistence measure only considers the difference in function value between the minimum and the saddle, and hence regards only the deepest point of the feature line.

\subsection{Feature Line Preserving Cancellation}

A feature line preserving cancellation criterion should quantify the whole feature line that is deleted by a cancellation. We want to keep just salient 1-cells from the initial MS complex which means that the quantification of the feature line should be compared to the vicinity of the line. The MS complex allows us both. An ascending 1-cell lies between two valleys which we use as vicinity. In the following, $I$ stands for an arbitrary scalar feature indicator. 


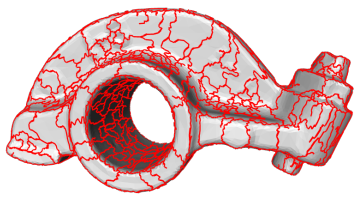

(a) Initial $\kappa_{1}$ MS complex.

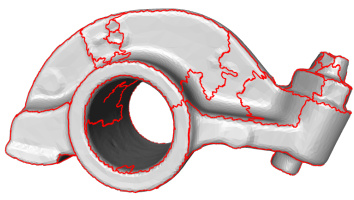

(b) Persistence, step 3,600.

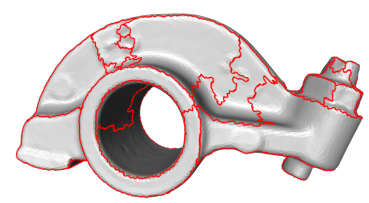

(c) Persistence, step 3,650.

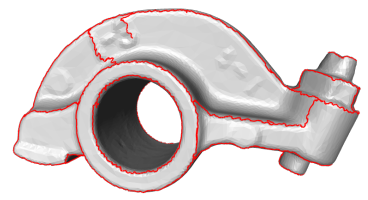

(d) Our method, step 3,550.

Figure 3: Ascending 1-cells of the initial MS complex of $\kappa_{1}$ on the rocker arm are a candidate set of convex feature lines in (a). 3,600 persistence cancellations lead to the line set depicted in (b). Clearly, all feature lines are still contained. After 3,650 cancellations, one of the main features is broken by persistence cancellation in (c). In contrast, feature line preserving cancellation as proposed in this paper maintains the main features in $(d)$.

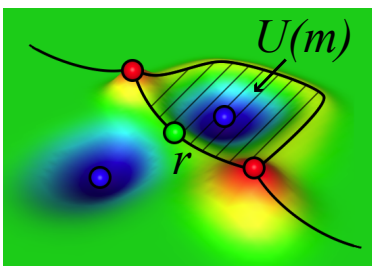

(a) Salient ascending 1-cell.

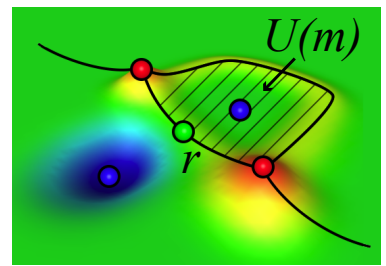

(b) Shallow ascending 1-cell.

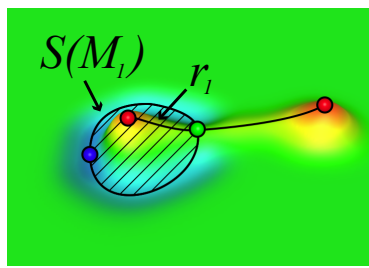

(c) Salient terminal 1-cell.

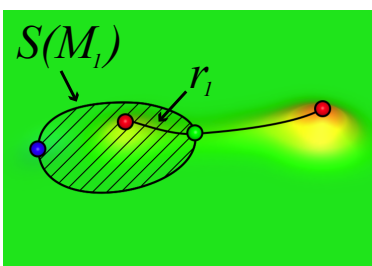

(d) Shallow terminal 1-cell.

Figure 4: Quantification of 1-cell deletion: the average curvature on the 1-cell is compared to the average curvature on the shaded area, and 1-cells with a larger difference are considered more salient, i.e., they are canceled later.

The proposed quantification $\sigma_{r}(s, m)$ of a cancellation of saddle $s$ and minimum $m$ deleting the ascending 1-cell $r$ is the difference of the average of $I$ on the 1-cell and the average of $I$ on the valley $U(m)$ containing $m$ :

$$
\sigma_{\max }(r, m)=\frac{1}{\operatorname{area}(r)} \int_{r} I-\frac{1}{\operatorname{area}(U(m))} \int_{U(m)} I,
$$

see Fig. 4a-b and analogously for cancellations with a maximum $M$ on the hill $S(M)$ deleting the descending 1-cell $v$,

$$
\sigma_{\min }(v, M)=\frac{1}{\operatorname{area}(S(M))} \int_{S(M)} I-\frac{1}{\text { length }(v)} \int_{v} I .
$$

A line can not be regarded as a feature if the accumulated $I$ average does not differ significantly compared to both incident patches. Hence, the feature line significance $\sigma_{\max }(r)$ and $\sigma_{\min }(v)$ is defined as the minimum of both values:

$$
\begin{aligned}
& \sigma_{\max }(r)=\min \left(\sigma_{\max }\left(r, m_{1}\right), \sigma_{\max }\left(r, m_{2}\right)\right), \\
& \sigma_{\min }(v)=\min \left(\sigma_{\min }\left(v, M_{1}\right), \sigma_{\min }\left(v, M_{2}\right)\right),
\end{aligned}
$$

where $r$ lies between the minima $m_{1}, m_{2}$, and $v$ between the maxima $M_{1}, M_{2}$. In words, a 1-cell is regarded as salient if on the line more average $I$-value is accumulated than in the patches that the 1-cell bounds.

Quantifying 1-cell cancellation as in (3), (4) preserves feature lines, but also, short offshoots of the feature lines are kept that fork off orthogonally from the requested lines. To punish such offshoots, $\sigma_{\max }, \sigma_{\min }$ are adjusted at terminal 1cells only. These emanate from those saddles, where one of the incident extrema is connected to just one saddle. For an ascending 1-cell $r$ with $r_{1}, r_{2}$ being the part of the 1-cell ascending to the maxima $M_{1}, M_{2}$, and a minimal 1-cell $v$ with $v_{1}, v_{2}$ being the part of the 1-cell descending to the minima $m_{1}, m_{2}$, respectively, the quantifications

$$
\begin{aligned}
\Gamma_{\max }\left(r_{i}, M_{i}\right) & =\frac{1}{\operatorname{area}\left(r_{i}\right)} \int_{r_{i}} I-\frac{1}{\operatorname{area}\left(S\left(M_{i}\right)\right)} \int_{S\left(M_{i}\right)} I \\
\Gamma_{\min }\left(v_{i}, m_{i}\right) & =\frac{1}{\operatorname{area}\left(U\left(m_{i}\right)\right)} \int_{U\left(m_{i}\right)} I-\frac{1}{\operatorname{length}\left(v_{i}\right)} \int_{v_{i}} I,
\end{aligned}
$$

see Fig. 4c-d are considered additionally by updating $\sigma_{\max }(r)$ and $\sigma_{\min }(v)$

$$
\begin{aligned}
\sigma_{\max }(r) & \leftarrow \min \left(\sigma_{\max }(r), \Gamma_{\max }\left(r_{1}, M_{1}\right), \Gamma_{\max }\left(r, M_{2}\right)\right), \\
\sigma_{\min }(v) & \leftarrow \min \left(\sigma_{\min }(v), \Gamma_{\min }\left(v, m_{1}\right), \Gamma_{\min }\left(v, m_{2}\right)\right) .
\end{aligned}
$$

In words, terminal feature lines that are not salient compared to the patches they lie in, are discarded as well. See Fig. 5 for an example of a 1-cell forking off a feature line.

Only valid cancellations may be performed to keep the alternating quad property of the MS complex. This means, a saddle/maximum cancellation is allowed only if the saddle connects two different maxima. Therefore we define the topological adaptations $t_{\max }(r)=\sigma_{\max }(r)$, if the 1-cell $r$ can be deleted by a valid cancellation, $\infty$ otherwise, and $t_{\min }(v)=\sigma_{\min }(v)$, if a valid cancellation exists, $\infty$ otherwise.

The cancellation criterion $t$ quantifying a saddle $s$ by the least significant cancellable 1-cell passing through the saddle is then defined by

$$
t(s)=\min \left(t_{\max }(r), t_{\min }(v)\right) .
$$

The reduction of lines in the initial complex now proceeds by canceling saddles in increasing order of $t$. This ensures that the lines with strongest feature line significance are kept. The saddle is canceled with the extremum for which equality is obtained in (9). See the result of using feature line significance as cancellation criterion in Fig. 3d. The deviation of 


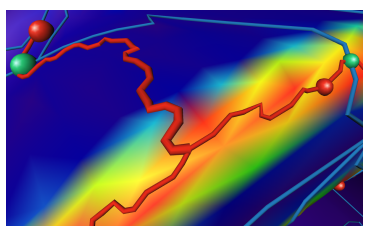

(a) Before cancellation.

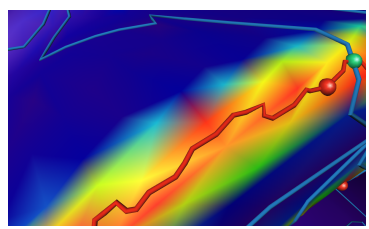

(b) After cancellation
Figure 5: Cancellation of a terminal 1-cell forking off the outer ring feature of the rocker arm colored by $\kappa_{1}$. The 1cell emanating from the left saddle ends at the maximum on the high curvature area, but only the part up to the branching point is being deleted by the cancellation.

the 1-cell as for persistence cancellation does not occur here; it stays on the feature line.

Two notes are important here:

1. Only one type of 1-cell is a meaningful feature line for most scalar indicators. We handle both types of 1-cells equally anyway for each quantity as this ensures a homogeneous cancellation of minima and maxima.

2. The 1-cell $r$ used to evaluate (1) is restricted to the path uniquely associated with the saddle under consideration. Paths from different saddles can merge before reaching the maximum as depicted in Fig. 5a. This is different from the smooth setting where different 1-cells never meet. In Fig. 5b, the left saddle was canceled with the terminal maximum. Only the unique part of the 1-cell was removed. The remaining path to the maximum connects to other saddles and is therefore retained. Hence, 1-cells are quantified up to the first branching point only.

\subsection{Discarding Topologically Forced Lines}

1-cells $r$ for which no valid cancellation exist might have a smaller feature line significance than the last canceled 1cell. In this case, $r$ is marked invisible and not displayed afterwards. Examples are the two concentric rings around the hole of the rockerarm in Fig. 3d that are not connected as forced by topology, and the cow's head in Fig. 9b, where the eye area is detached from all other lines.

\subsection{Optional Line Smoothing}

Our method results in lines that either consist of vertices or triangle centers of the mesh. While this is topologically correct, it might not be visually appealing. We applied a simple averaging with fixed filter length. The adapted Laplacian line smoothing technique introduced by Hildebrandt et al [HP04] gives appropriate guarantees when needed.

\subsection{Implementational Issues}

For the computation of the MS complex hierarchy we use the approach introduced by Cazals et al [CCL03]. Instead of using the Union Find structure detailed there, we work directly on the primal and dual spanning tree. A saddle / minimum cancellation is performed by adding the saddle as edge into the primal spanning tree and flipping all edges on the path from the saddle to the cancelled minimum. This results in simple algorithms but affects the performance as for each cancellation a number of edge flips have to be performed.

For computing feature line significance $t$ (9), the involved quantities are stored in the primal and dual spanning trees. Each minimum and each maximum is being initialized with the area of their unstable and stable manifolds respectively, and with the integral of the scalar indicator over the manifold. Any saddle stores the integral and area of the stable manifolds (triangle strips), and the integral and length of the unstable manifolds (edge sequence) emanating from the saddle. Upon cancellation, those values are propagated from the canceled extrema to its uncanceled analogon, so computation of feature line significance requires $O(1)$ time.

All saddles are stored in an AVL tree that is used as priority queue. When a saddle is canceled, the saddles incident to its incident extrema have to be reevaluated, as $t$ can be reduced by a cancellation. In theory, a single extremum can be incident to a majority of the saddles in the complex which means $O(n \log n)$ for a single update of the queue in the worst case. In practice, $t$ is designed to cancel minima and maxima on the same priority, and hence our cancellation criterion is not significantly slower than persistence cancellation.

\section{Results}

For all results, the used curvature estimates are computed with the discrete method introduced by Hildebrandt et al [HPW06], without smoothing. All feature lines are shown as extracted by the method. We use red lines to display convex features (maximal lines of $\kappa_{1}$ ), blue lines for concave features ( $\kappa_{2}$ minimal), black lines for curvedness $C$. Smoothed lines are displayed in the unshaded images only, all others contain the unsmoothed originals.

Fig. 3 shows the rockerarm surface with $20 K$ triangles. The initial ascending 1-cells are shown together with the result of our method after 3,550 (of total 3,875) cancellations. The extraction of the concave features is displayed analogously in Figure 6. Displaying both sets of feature lines in Figure $6 \mathrm{~d}$ represents the geometry of the rockerarm well.

Treating concave and convex features separately results in two parameters. Curvedness allows us to reduce this to one parameter, as the maximal structures of curvedness contain both convex and concave features lines, as displayed in Fig. 7. The ascending 1-cells of the MS complex corresponding to the optimal cancellation value 3,388 (out of total 3,777 ) in Figures 7b-c represent the geometry of the surface, dependent on just one parameter. Due to this, curvedness is particularly suitable for our approach and we favor it over the linked usage of $\kappa_{1}$ and $\kappa_{2}$, unless either convex or concave features are of particular interest for a specific surface, as for the Stanford bunny with $280 \mathrm{~K}$ triangles in Fig. 8, where the concave features alone reflect the geometry well. In this case, the initial descending 1-cells of $\kappa_{2}$ are densely spread 


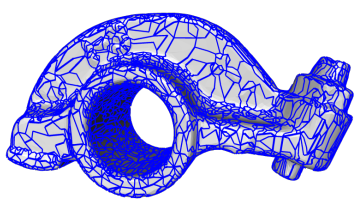

(a) Initial $\kappa_{2}$ MS complex.

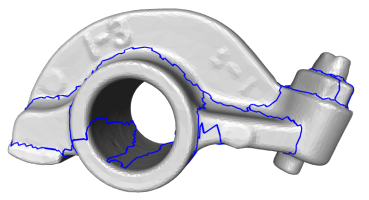

(b) Our method, step 4460.

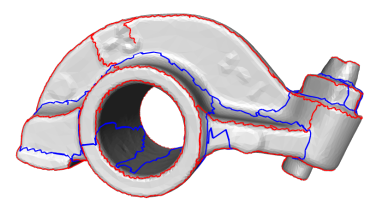

(c) Concave and convex lines.

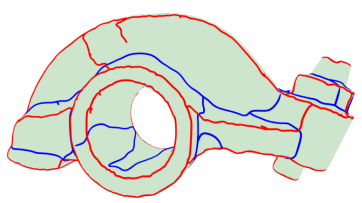

(d) Features plus silhouettes.

Figure 6: a) The initial MS complex of $\kappa_{2}$ containing 4476 descending 1-cell is taken as a starting point. b) 4460 feature preserving cancellations yield the visually best concave feature lines. $c, d$ ) Feature lines plus silhouette represent the geometry.

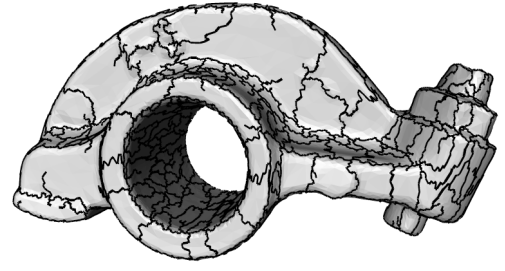

(a) Initial Curvedness MS complex.

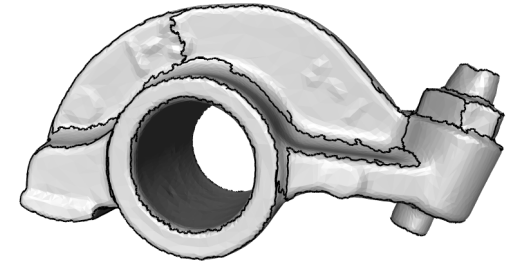

(b) Feature lines of curvedness.

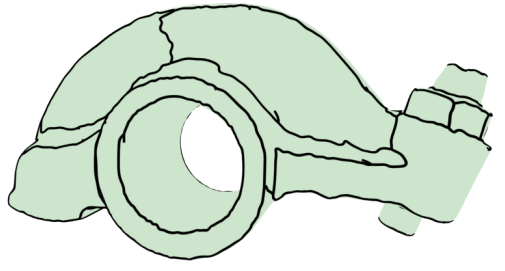

(c) Features plus silhouettes.

Figure 7: The extraction of maximal features of the Curvedness indicator yields both convex and concave feature lines in one. It depends on just one parameter, compared to two for the linked treatment of $\kappa_{1}$ and $\kappa_{2}$ and is therefore our preferred technique.

over the whole surface. The most significant concave feature lines are displayed in Figure 8d. In Fig. 9 the feature lines on the cow's head consisting of $93 \mathrm{~K}$ triangles are displayed based on curvedness. Three different feature scales corresponding to increasing cancellation values are being displayed, ranging from fine scale in Figures 9a,b) to low scale in Figure d). It is interesting to see that the head can be recognized at quite coarse scale, and how the addition of fine scale features adds expression to the cow's face.

Fig. 9d shows another property of our approach: The terminal 1-cell building the cow's brow is no watershed (as it does not separate two regions) and could only be extracted, as the whole MS complex is used for feature extraction.

The computational complexity of our approach is $n \log n$ for the extraction of the initial MS complex (see [CCL03]), where $n$ is the number of vertices of the mesh. The current implementation requires the 1-cells to be updated directly on the graphs, which is of order $n$ for a single cancellation in the worst case. Table 1 gives timings of our method measured on a workstation with an Intel Xeon CPU with $3.00 \mathrm{GHz}$ and 16 GB Ram without parallelization. We have not put much emphasis on efficiency, and it can certainly be improved.

\subsection{Insensitivity to Noise}

Our method is insensitive to noise in the surface mesh. To show this, we add Gaussian noise to the vertex positions of the rockerarm mesh in direction to the mesh normal. We used $\sigma=\frac{1}{5}, \sigma=\frac{1}{2}, \sigma=1$ of the mean edge length. Those values correspond to the significant deformations seen in Fig. 10, but the extracted Curvedness feature lines are still visually close to the Curvedness feature lines extracted from the undistorted mesh in Figure 7.

\begin{tabular}{lcccc}
\hline surface & indicator & \#cancels & $t_{\text {construct }}$ & $t_{\text {cancel }}$ \\
\hline \hline rocker arm & $\kappa_{1}$ & 3,678 & 0.21 & 0.50 \\
\hline & $\kappa_{2}$ & 4,476 & 0.23 & 0.55 \\
\hline bunny & $C$ & 3,777 & 0.20 & 0.44 \\
\hline cow & $\kappa_{2}$ & 71,577 & 3.8 & 30 \\
\hline vase-lion & $C$ & 13,676 & 1.2 & 7.6 \\
\hline \hline
\end{tabular}

Table 1: Timings of our method for various datasets and scalar feature indicators. In rows 3-5, the number of cancellations, the time in seconds to construct the initial MS complex, and for performing all cancellations are shown.

\subsection{User Guided Parameter Traversal}

When using Curvedness as feature indicator, our method depends only on one parameter. This parameter is easy to control, as it allows for binary search. As the initial feature set usually contains far too many lines, we proceed by performing two thirds of the remaining cancellations successively, until visually too few feature lines are contained. In most cases, the previous line set is then very close to the optimum. At $\operatorname{most} \log n$ values of the cancellation parameter have to be tried, where $n$ is the number of cancellations. An example can be seen in Fig. 11 for the lion vase dataset consisting of about $78 K$ triangles allowing for 17,613 cancellations.

\subsection{Limitations}

The proposed method has two limitations. Firstly, it results in long connected lines. Whereas this is advantageous in some settings, it is too rigid in others, as it is the case on the rocker arm surface, where our technique fails to resolve the "1-3"-Feature on the side of the rocker arm both in the $\kappa_{1}$ case (Fig. 3), and in the $C$ case (Figure 7). Secondly, as 


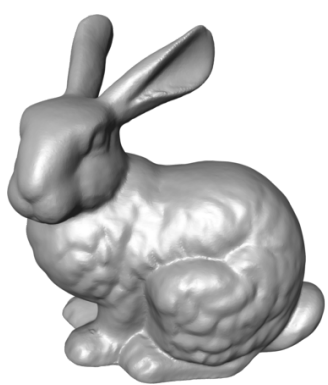

(a) The Stanford bunny

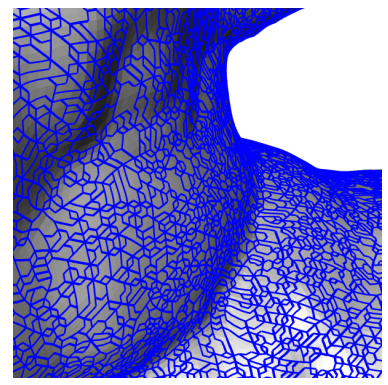

(b) Initial $\kappa_{2}$ MS complex.

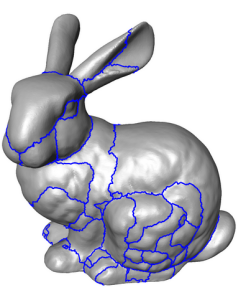

(c) Step 71,440.

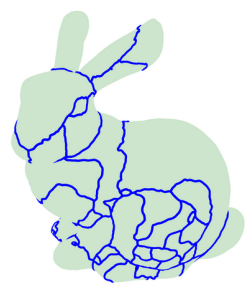

(d) Features plus silhouettes.

Figure 8: Concave feature lines are extracted on the Stanford bunny with $280 \mathrm{~K}$ triangles. The initial MS complex of $\kappa_{2}$ consists of 71,577 cancellations, and its descending 1-cells are densely spread over the bunny. The kept concave features identify it.

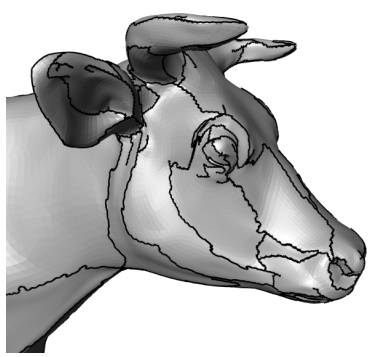

(a) Fine scale features, shaded.

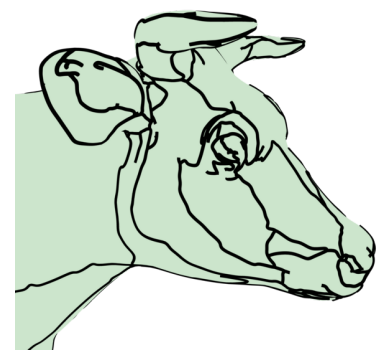

(b) Fine scale features.

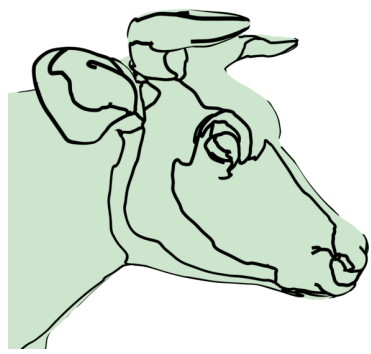

(c) Middle scale features.

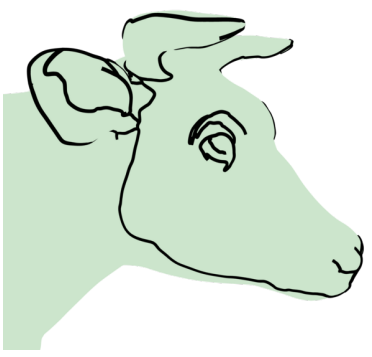

(d) Coarse scale features.

Figure 9: Feature lines on the cow at fine, medium and coarse scale after 12,451, 13,116 and 13,491 cancellations respectively. The coarse scale suffices to reflect the geometry, and fine scale features add more and more expression to the cow's face.

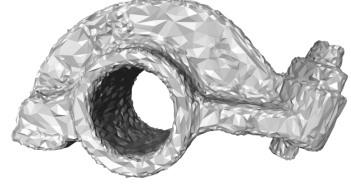

(a) Surface at $\sigma=\frac{1}{5}$.

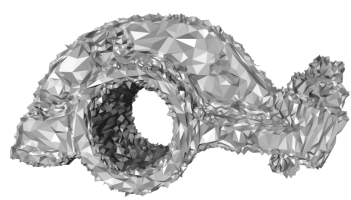

(c) Surface at $\sigma=\frac{1}{2}$.

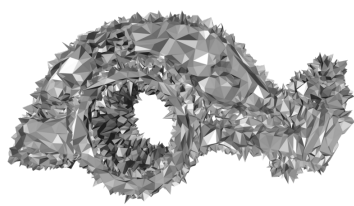

(e) Surface at $\sigma=1$.

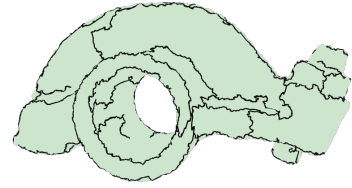

(b) Features at $\sigma=\frac{1}{5}$

(d) Features at $\sigma=\frac{1}{2}$

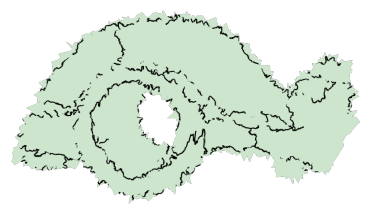

(f) Features at $\sigma=1$

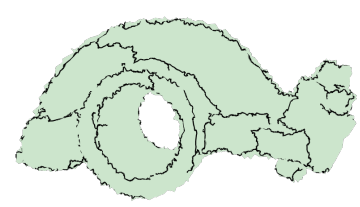

Figure 10: Adding Gaussian noise scales to the rockerarm shows the stability of our method. The feature lines just appear disconnected due to occlusion by surface spikes. mentioned in the last paragraph of Sect. 3.7, our cancellation criterion $t$ from (9) is not monotone, i.e., it can be smaller at later cancellation stages. This makes it impossible to specify a cancellation threshold rather than a cancellation count.

\section{Conclusions}

We showed how the Morse-Smale complex of scalar feature indicators and an appropriately adapted cancellation criterion can be used to extract meaningful feature lines of surfaces in a stable way, dependent on just one parameter. In this setting, we identified Curvedness as the most suitable feature indicator for the extraction of surface features.

In the future we aim at comparing the feature lines of our technique with ridge lines, both on a theoretical and on a practical level. Further research directions are twofold: an optional post-processing step seems appropriate in cases, where long connected feature lines are not desired. Furthermore, after having reduced the number of necessary parameters for feature line extraction to one, parameter free feature line extraction seems within reach, e.g. by examining cancellations statistics to provide the user with an initial guess for an optimal cancellation parameter.

\section{Acknowledgements}

The rocker arm and the cow's head are courtesy of INRIA, the vase-lion of SenSable Technologies inc., provided by Aim@Shape. The bunny is courtesy of the Stanford Scanning Repository. 

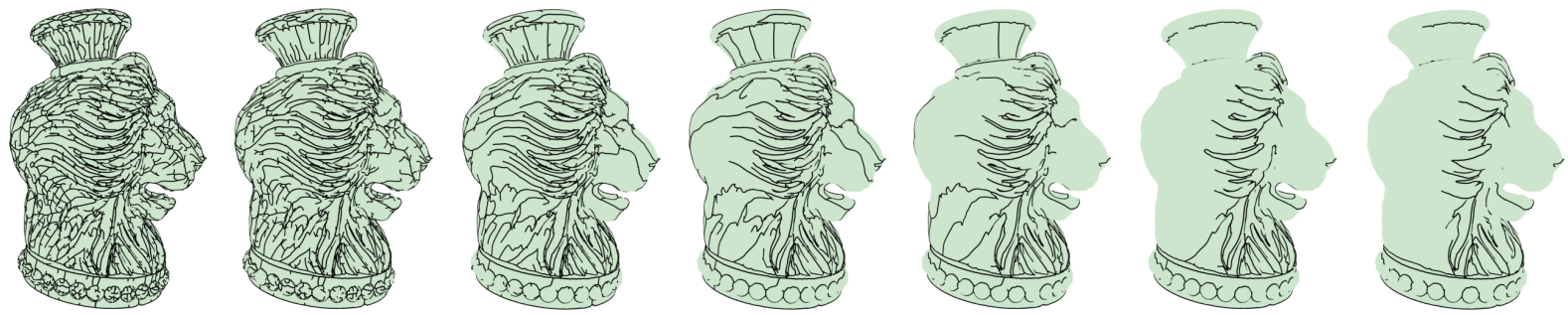

Figure 11: Feature lines on the lion vase. The one parameter our method depends on is traversed logarithmically. A user has to decide for less lines until the coarse level of detail he is interested in is reached. One further step removes a detail regarded as interesting. This way a good overview of the various level of details contained in the dataset is acquired in a few steps.

\section{References}

[Ban70] BANCHOFF T.: Critical points and curvature for embedded polyhedral surfaces. Am. Math. Monthly (1970), 475-485.

[BeHP04] Bremer P., Edelsbrunner H., Hamann B., PASCUCCI V.: A topological hierarchy for functions on triangulated surfaces. IEEE TVCG 10, 4 (2004), 385-396.

[CCl03] Cazals F., Chazal F., Lewiner T.: Molecular shape analysis based upon the morse-smale complex and the connolly function. In SCG: Proc. of the 19. Ann. Symp. on Comp. Geom. (2003), pp. 351-3605.

[DBG*06] Dong S., BRemer P.-T., Garland M., Pascucci V., HART J. C.: Spectral surface quadrangulation. In $S I G$ GRAPH (2006), ACM Press, pp. 1057-1066.

[Ebe96] EBERLY D.: Ridges in Image and Data Analysis. Kluwer Acadamic Publishers, Dordrecht, 1996.

[EHZ03] Edelsbrunner H., Harer J., Zomorodian A.: Hierarchical morse-smale complexes for piecewise linear 2manifolds. In Disc. Comput. Geom. (2003), vol. 30, pp. 87-107.

[ELZ00] Edelsbrunner H., Letscher D., Zomorodian A.: Topological persistence and simplification. In FOCS '00: Proc. of the 41st Ann. Symp. on Foundations of Computer Science (Washington, DC, USA, 2000), IEEE Computer Society, p. 454.

[For98] FORMAn R.: Morse theory for cell complexes. Advances in Mathematics 134 (1998), 90-145.

[For02] FORMAN R.: A user's guide to discrete morse theory. Sémin.Lothringien de Combinatoire B48c (2002).

[GMDP*07] Gyulassy A., M. Duchaineau V. N., PasCucci V., Bringa P., Higginbotham A., Hamann B.: Topologically clean distance fields. In Proc. IEEE Visualization 2007 (2007), pp. 1432-1439.

[HP04] Hildebrandt K., Polthier K.: Anisotropic filtering of non-linear surface features. Comput. Graph. Forum 23, 3 (2004), 391-400.

[HPW05] Hildebrandt K., Polthier K., Wardetzky M.: Smooth feature lines on surface meshes. In 3rd Eurographics Symp. on Geom. Proc. (2005), pp. 85-90.

[HPW06] Hildebrandt K., Polthier K., Wardetzky M.: On the convergence of metric and geometric properties of polyhedral surfaces. Geometria Dedicata 123 (2006), 89-112.

[KvD92] Koenderink J. J., van Doorn A. J.: Surface shape and curvature scales. Image and Vision Computing 10, 8 (October 1992), 557-564.
[Lew05] LEwiner T.: Mesh Compression from Geometry. Thèse de doctorat, Université Pierre et Marie Curie, France, 2005.

[LlT03a] Lewiner T., Lopes H., TAvares G.: Optimal discrete morse functions for 2-manifolds. Comput. Geom. Theory Appl. 26, 3 (2003), 221-233.

[LlT03b] Lewiner T., Lopes H., Tavares G.: Visualizing Forman's discrete vector field. In Visualization and Mathematics III, Hege H.-C., Polthier K., (Eds.). "Springer-Verlag, Heidelberg, 2003, pp. 95-112

[Mil63] MiLnOR J. W.: Morse Theory, vol. 51 of Annals of Mathematics Studies. Princeton, NJ, USA, 1963.

[MW99] MANGan A. P., WhitaKer R. T.: Partitioning 3d surface meshes using watershed segmentation. IEEE TVCG 5, 4 (1999), 308-321.

[NWB*06] Natarajan V., Wang Y., Bremer P. T., PasCUCCI V., HAMANN B.: Segmenting molecular surfaces. Computer Aided Geometric Design (special issue on Applications of Geometric Modeling in the Life Sciences) 23, 6 (2006), 495-509.

[OBS04] OhtaKe Y., BelYaev A., SeIDEl H.-P.: Ridge-valley lines on meshes via implicit surface fitting. In SIGGRAPH (2004), pp. 609-612.

[Pas07] PAscucci P.-T. B. V.: A practical approach to twodimensional scalar topology. In Topology-based Methods in Visualization, Hauser H., Hagen H., Theisel H., (Eds.), Mathematics and Visualization. Springer, 2007.

[PM82] Palis J., Melo W. D.: Geometric Theory of Dynamical Systems: An Introduction. Springer-Verlag, 1982.

[SF04] Stylianou G., FARIN G.: Crest lines for surface segmentation and flattening. IEEE Transactions on Visualization and Computer Graphics 10, 5 (2004), 536-544.

[Soi99] SolLle P.: Morphological Image Analysis. Springer, Berlin, Heidelberg, New York, 1999.

[SWTH07] Sahner J., Weinkauf T., Teuber N., Hege H.C.: Vortex and strain skeletons in eulerian and lagrangian frames. IEEE TVCG 13, 5 (2007), 980-990.

[YBS05] Yoshizawa S., Belyaev A., Seidel H.-P.: Fast and robust detection of crest lines on meshes. In Proc. ACM SPM (2005), pp. 227-232.

[YBYS07] Yoshizawa S., Belyaev A. G., Yokota H., SeIDEL H.-P.: Fast and faithful algorithm for detecting crest lines on meshes. In Pacific Graph. (2007). 